University of Nebraska - Lincoln

DigitalCommons@University of Nebraska - Lincoln

2012

\title{
Transmission electron microscopy of single wall carbon nanotube/polymer nanocomposites: A first-principles study
}

\author{
F. Solá \\ NASA Glenn Research Center, francisco.sola-lopez@nasa.gov
}

Z. H. Xia

University of North Texas, Zhenhai.Xia@unt.edu

M. Lebrón-Colón

NASA Glenn Research Center, Lebron-Colon-1@nasa.gov

M. A. Meador

NASA Glenn Research Center, maryann.meador@nasa.gov

Follow this and additional works at: https://digitalcommons.unl.edu/nasapub

Solá, F.; Xia, Z. H.; Lebrón-Colón, M.; and Meador, M. A., "Transmission electron microscopy of single wall carbon nanotube/polymer nanocomposites: A first-principles study" (2012). NASA Publications. 123. https://digitalcommons.unl.edu/nasapub/123

This Article is brought to you for free and open access by the National Aeronautics and Space Administration at DigitalCommons@University of Nebraska - Lincoln. It has been accepted for inclusion in NASA Publications by an authorized administrator of DigitalCommons@University of Nebraska - Lincoln. 


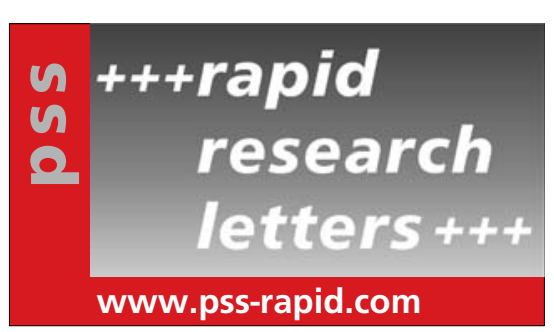

\title{
Transmission electron microscopy of single wall carbon nanotube/polymer nanocomposites: A first-principles study
}

\author{
F. Solá*, , Z. H. Xia ${ }^{2}$, M. Lebrón-Colón', and M. A. Meador ${ }^{1}$ \\ ${ }^{1}$ Materials and Structures Division, NASA Glenn Research Center, Cleveland, OH 44135, USA \\ ${ }^{2}$ Department of Materials Science and Engineering, University of North Texas, Denton, TX 76203, USA
}

Received 23 June 2012, revised 1 August 2012, accepted 6 August 2012

Published online 8 August 2012

Keywords transmission electron microscopy, multislice calculations, molecular dynamics simulations, polymer nanocomposites

* Corresponding author: e-mail francisco.sola-lopez@nasa.gov, Phone: +1 216433 6954, Fax: +1 2169777132

The physics of high resolution transmission electron microscopy (HRTEM) image formation and electron diffraction of single wall carbon nanotubes (SWCNTs) in a polymer matrix was investigated theoretically on the basis of the multislice method. The effect of the nanocomposite thickness on both image contrast and typical electron diffraction reflections of the nanofillers was explored. The implications of the results on the experimental applicability to study dispersion, chirality and diameter of nanofillers are discussed.
Polymer nanocomposites filled with one-dimensional (1D) carbon based nanostructures are potential candidates for aerospace applications [1]. In particular, single wall carbon nanotubes (SWCNTs) have advantages over conventional reinforcement materials, like carbon fiber, because of their unique properties [2]. There are several factors to take into consideration in order to optimize the nanocomposite electrical, mechanical and thermal properties. One is the ability to homogeneously disperse the nanotubes into the polymeric material. This has been proved to play an important role to improve each one of the previous mentioned nanocomposite properties [2]. For instance, the interface between the nanotubes and polymer matrix is optimized if the nanotubes are well dispersed, which improves the mechanical response of the nanocomposite. In contrast, nanotubes dispersed as aggregates in the polymer matrix will diminish the interaction at the interface [3]. In the case of electrical properties, although dispersion is important, the major control has to be focused on a reliable method to identify an abundance of metallic/ semiconducting type of nanotubes through chirality [4].

Transmission electron microscopy (TEM) is a technique regularly used to characterize the dispersion of nanofillers in a polymeric matrix. However, imaging SWCNTs in a polymer matrix is challenging due to both its small size (typical diameter $\sim 10 \AA$ ) and that its contrast is covered by scattering from the polymer matrix. In this work, we are using modeling results based on high resolution TEM (HRTEM) imaging and electron diffraction (ED) for a deeper analysis of polymer nanocomposites. The implications of our results on the dispersion of SWCNTS in a polymer matrix, as well as chirality determination will be discussed.

Molecular dynamics (MD) was used to simulate SWCNTs and their corresponding interactions with a polyethylene (PE) matrix. The interactions between atoms were calculated using an adaptive intermolecular reactive empirical bond-order (AIREBO) potential, coupled to a longrange Lennard-Jones potential [5]. With a rescale thermostat to control temperature, the equations of motion were integrated with a time step of $0.5 \mathrm{fs}$ at $300 \mathrm{~K}$. Ideal SWCNT configuration was first generated with the graphitic $\mathrm{C}-\mathrm{C}$ bond length of $1.42 \AA$. A nanotube diameter of $d=10.59 \AA$ with a length of $L=50 \AA$ was used in all calculations. Finally, polyethylene matrices with different thicknesses were generated with Materials Studio. A hole of some diameter, approximately $6 \AA$ larger than that of the nanotube, is drilled out of the center of the matrix sample, and a nanotube is inserted into it. The system was then relaxed to equilibrium under zero applied load. The 
SWCNT/matrix interface is controlled via van der Waals forces and no chemical bonding exists across the interface in this study. Figure 1a is the cross sectional model of the SWCNT/PE nanocomposite, along with the top view of the isolated SWCNT.

HRTEM and ED modeling was performed by the standard multislice theory [6], with a slice width of $2 \AA$. One advantage of the multislice method is that both crystalline and amorphous materials can be simulated. The transfer function (TF) used here contains parameters to account for defocus $(\Delta f)$, third-order spherical aberration $\left(C_{\mathrm{s}}\right)$, and damping envelope functions to describe partial temporal and spatial coherence. Other aberrations such as astigmatism and coma are not considered in this work. For the computations, we used the parameters of an FEG Super TWIN Philips CM 200 TEM operated at $80 \mathrm{kV}$. That is, both coefficients of chromatic aberration and $C_{\mathrm{s}}$ have values of $1.2 \mathrm{~mm}$, while the energy spread and the spread angle have values of $0.8 \mathrm{eV}$ and $0.05 \mathrm{mrad}$, respectively [7]. The optimum defocus or Scherzer defocus is obtained as $\Delta f=-1.33\left(\lambda C_{\mathrm{s}}\right)^{0.5}$, where $\lambda$ is the wavelength of electrons accelerated at $80 \mathrm{kV}$. There are two reasons for having chosen this accelerating voltage. First, this accelerating voltage is below the knock-on damage of SWCNTs [8]. Second, at this accelerating voltage the electrons do not experience relativistic effects; therefore, the use of the Schrödinger equation in our simulations is strictly valid. ED patterns used a $0.5 \mathrm{mrad}$ convergent beam. Figure $1 \mathrm{~b}$ corresponds to the TF used in this work at Scherzer defocus, where the typical high spatial frequency features are highly oscillatory and damped. It is the high spatial frequencies that are important in HRTEM.

Figure 1c is a HRTEM image of a SWCNT/PE nanocomposite with an effective thickness of $25 \AA$. The inset corresponds to the results of the isolated SWCNT. There

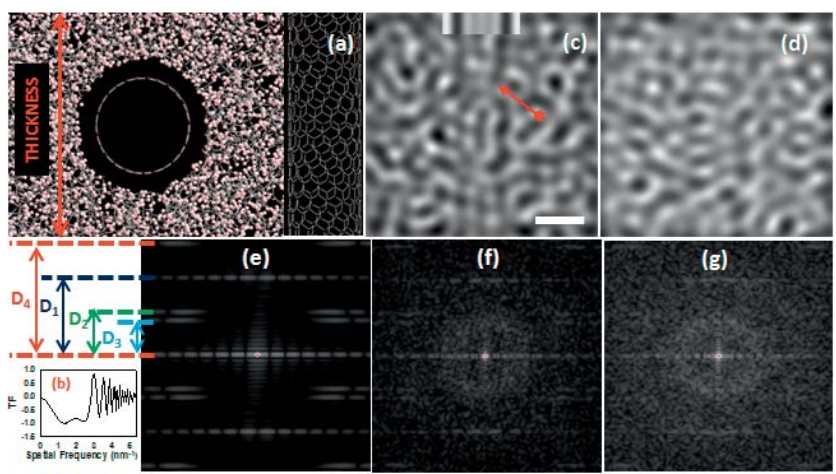

Figure 1 (online color at: www.pss-rapid.com) Model of SWCNT/PE nanocomposite (a), transfer function (b), and HRTEM images (scale bar is $10 \AA$, Scherzer defocus) for a nanocomposite of thickness $25 \AA$ (c) and $49 \AA$ (d), respectively. The inset in (c) is a HRTEM of the isolated nanotube. ED of pristine (e), $25 \AA$ (f) and $49 \AA$ (g) nanocomposite, respectively. ED was taken with a $0.5 \mathrm{mrad}$ convergent beam. For both HRTEM and ED results, the nanotube walls were positioned perpendicular to the electron beam. For more details see the text. are several contrast features that are different in the images. Firstly, there is an apparent reduction in both the dark contrast intensity and width of the walls of the nanotube located in the polymer matrix relative to the free standing nanotube. Secondly, the walls of the nanotube cannot be completely resolved for the case of the nanocomposite. In addition, there are some features (see the red arrow) in the walls of the nanotube that can be misinterpreted as defects created from the formation of pentagonal rings. Note that the SWCNT in this work is a perfect, defect free nanotube. Therefore these features clearly come from the polymer matrix. The situation becomes worse with an increase in thickness of the nanocomposite. As can be seen in Fig. 1d, for a nanocomposite of $49 \AA$ the nanotube walls disappear completely in the HRTEM image. Similar results have been observed in SWCNT positioned at the top surface of an amorphous carbon film [9]. However, in the ED pattern for this nanocomposite (see Fig. 1g), some typical reflections of carbon nanotube are visibly superimposed by the amorphous background from the polymer matrix. This information can be used to extract diameter information as well as determine if the nanotube is metallic or semiconducting. An SWCNT can be formed by wrapping a single sheet of graphene, which is described by a vector with components $(n, m)$. Once having the $(n, m)$ vector, the nanotube diameter $(d)$ can be computed as $d=2.461 \AA$ $\times\left(n^{2}+m^{2}+n m\right)^{0.5} / \pi$ [10]. Furthermore, if $n-m=3 j(j$ is an integer different from zero) the nanotube is metallic, otherwise it is a semiconductor [10]. Regarding the ED of SWCNT, $(n, m)$ can be extracted from the ratio of the distances of horizontal lines relative to the equatorial line [10]. Figure 1e is the ED for the free standing SWCNT, and the inset corresponds to the line distances relative to the equatorial line. Only a portion of the whole ED pattern is shown, to focus in the first four lines. The distance values are $D_{1}=0.4639 \AA^{-1}, D_{2}=0.2577 \AA^{-1}, D_{3}=0.2062 \AA^{-1}$ and $D_{4}=0.6701 \AA^{-1}$, respectively. The ratio $m / n=$ $\left(3 D_{1} / D_{4}\right)-1$, can be used to extract the chiral vector $(13,1)$.

For the nanocomposite of thickness $25 \AA$, the features from all four lines are still visible and the amorphous background has not considerably affected the reflections of the lines. Increasing the thickness of the nanocomposite to $49 \AA$ has a strong effect on the diffraction reflections from the second and third lines, as can be seen in Fig. 1g. However, the first and fourth lines are still visible, and therefore the chiral vector $(13,1)$ and hence diameter of $10.59 \AA$ can be extracted accurately, even if the nanotube cannot be resolved in the HRTEM image. Naturally, the following valid and fundamental question arises: What is the nanocomposite thickness limit in which the electron diffraction reflections from the nanotube are completely covered by the amorphous background of the polymer matrix? An exact answer to this question is not known at the moment. However, we have performed simulations for a nanocomposite of thickness $100 \AA$ and found that only a portion of diffraction reflections from the equatorial line were visible. 
Other reflections were covered by the polymer amorphous background. Clearly in this scenario the chirality and hence the diameter of the nanotube cannot be estimated, so there is a critical nanocomposite thickness in which our analysis may be useful.

There is another factor that may affect the ED pattern. The atoms of the structures analyzed here were fixed in space. Experimentally, TEM analysis is usually done close to room temperature, where the atoms thermally vibrate. Thermal vibrations can affect ED in a way that the background can increase by the so-called thermal diffuse scattering [11], which may reduce the intensity of the ED reflections from the nanotube. However, this effect can be reduced by using dedicated cooling holders. Consequently, the theoretical results presented here are more consistent with experiments performed using a cooling holder and may be useful perhaps in characterizing the dispersion of SWNCTs in the polymer matrix. Assuming that the ED can be fully or partially resolved, agglomeration of nanotubes as bundles can be identified since the electron diffraction reflections will form continuous arcs in this case [10]. A clear challenge to obtain this information from experiments is how to focus the electron beam to an area containing an SWCNT which is not visible in the image. A possible way to achieve this is to raster the beam slowly in the corresponding image area while at the same time collecting the ED for a given pixel in the image, using similar protocols developed recently to study diffraction along single nanowires [12].

We have also examined the effect of focusing strength on image contrast. Figure 2 summarizes the HRTEM im-

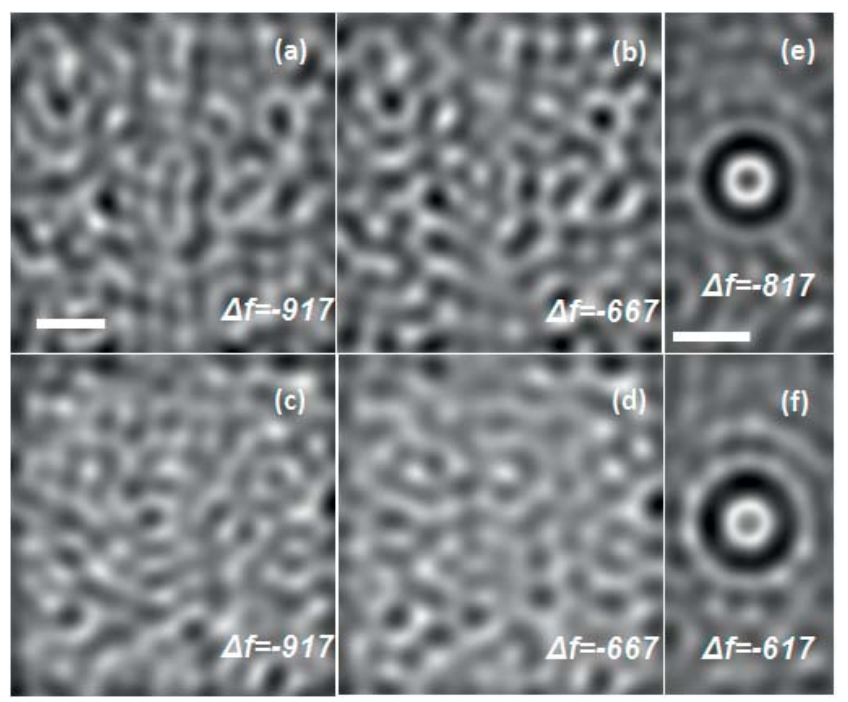

Figure 2 HRTEM images (scale bar is $10 \AA$ ) for a nanocomposite of thickness $25 \AA$ (a, b) and $49 \AA$ (c, d) respectively, where nanotube walls were positioned perpendicular to the electron beam. HRTEM images (scale bar is $10 \AA$ ) at different focus conditions for a nanocomposite of thickness $51 \AA$ at (e) Scherzer defocus and (f) $-617 \AA$, respectively. Nanotube walls were positioned parallel to the electron beam. Defocus value unit is $\AA$. ages taken at different focus conditions for nanocomposites of thickness $25 \AA$ (Fig. 2a, b) and $49 \AA$ (Fig. 2c, d), respectively. No clear improvement was obtained in regard to resolving the nanotube walls. All the data presented until now has the nanotube walls oriented perpendicular to the electron beam of the instrument. Figure 2e, f are HRTEM images of a nanocomposite of $51 \AA$ thickness taken at different defocus conditions with the nanotube walls parallel to the electron beam. Clearly the walls of the nanotube can be resolved. An apparent increase in nanotube wall thickness can be seen as the defocus value is increased, with diameter values of $12.1 \AA$ (Fig. 2e) and $12.9 \AA$, respectively. Therefore, even at optimum focus conditions (Fig. 2e), measuring the nanotube diameter will be an overestimation of about $1.5 \AA$. As a result, estimating the chirality from HRTEM images will be erroneous. For example, at the Scherzer defocus the nanotube diameter value corresponds to $(13,4)$ and not to the true $(13,1)$. As a final point, electron diffraction analysis is not useful in this case for the estimation of chirality or diameter.

In conclusion, we have investigated theoretically HRTEM images and electron diffraction of nanovolumes composed of PE filled with SWCNTs with the aim of obtaining diameter and chirality of the nanofiller, using multislice theory and the optics of an FEG TEM at $80 \mathrm{kV}$. With the electron beam oriented perpendicular to the walls of the nanotube, it was observed that the results strongly depend on the nanocomposite thickness and that HRTEM features of the nanofiller can completely disappear as the thickness increases, although chirality (and hence diameter) may be extracted by the first and second lines reflections of the electron diffraction pattern. Moreover, the metallic or semiconducting nature of SWCNTs can be established. By combining results from data collected with the beam parallel and perpendicular to the walls of the nanotube, it may be possible to study the dispersion of SWCNTs in a polymer matrix at the nanoscale level for a better understanding of the nanocomposite properties. Limitations of this approach in its experimental applicability were discussed.

\section{References}

[1] J. Njuguna and K. Pielichowski, Adv. Eng. Mater. 6, 193 (2004).

[2] M. Lebrón-Colón et al., ACS Appl. Mater. Interfaces 2, 669 (2010).

[3] C. Velasco-Santos et al., Composite Interfaces 11, 567 (2005).

[4] Y. Chen et al., ACS Macro Lett. 1, 246 (2012).

[5] L. Pastewka et al., Phys. Rev. B 78, 161402 (2008).

[6] E. J. Kirkland, Advanced Computing in Electron Microscopy (Springer, New York, 2010), p. 137.

[7] M. T. Otten and W. M. Coene, Ultramicroscopy 48, 77 (1993).

[8] A. V. Krasheninnikov and K. Nordlund, J. Appl. Phys. 107, 071301 (2010).

[9] L.-C. Qin et al., Chem. Phys. Lett. 349, 389 (2001).

[10] L.-C. Qin, Phys. Chem. Chem. Phys. 9, 31 (2007).

[11] L. He and R. Hull, Nanotechnology 23, 205705 (2012).

[12] K. J. Ganesh et al., Microsc. Microanal. 16, 614 (2010). 